\title{
ON A CERTAIN HOLOMORPHIC CURVE EXTREMAL FOR THE DEFECT RELATION
}

\author{
Nobushige TodA
}

\section{Introduction}

Let $f=\left[f_{1}, \ldots, f_{n+1}\right]$ be a holomorphic curve from $\boldsymbol{C}$ into the $n$-dimensional complex projective space $P^{n}(\boldsymbol{C})$ with a reduced representation

$$
\left(f_{1}, \ldots, f_{n+1}\right): \boldsymbol{C} \rightarrow \boldsymbol{C}^{n+1}-\{\boldsymbol{0}\},
$$

where $n$ is a positive integer.

We use the following notations:

$$
\|f(z)\|=\left(\left|f_{1}(z)\right|^{2}+\cdots+\left|f_{n+1}(z)\right|^{2}\right)^{1 / 2}
$$

and for a vector $\boldsymbol{a}=\left(a_{1}, \ldots, a_{n+1}\right) \in \boldsymbol{C}^{n+1}-\{\mathbf{0}\}$

$$
\begin{aligned}
\|\boldsymbol{a}\| & =\left(\left|a_{1}\right|^{2}+\cdots+\left|a_{n+1}\right|^{2}\right)^{1 / 2}, \\
(\boldsymbol{a}, f) & =a_{1} f_{1}+\cdots+a_{n+1} f_{n+1}, \\
(\boldsymbol{a}, f(z)) & =a_{1} f_{1}(z)+\cdots+a_{n+1} f_{n+1}(z) .
\end{aligned}
$$

The characteristic function of $f$ is defined as follows (see [11]):

$$
T(r, f)=\frac{1}{2 \pi} \int_{0}^{2 \pi} \log \left\|f\left(r e^{i \theta}\right)\right\| d \theta-\log \|f(0)\| .
$$

Due to Cartan ([1]), we have the following relation:

$$
T(r, f)=\frac{1}{2 \pi} \int_{0}^{2 \pi} \log \max _{1 \leq j \leq n+1}\left|f_{j}\left(r e^{i \theta}\right)\right| d \theta+O(1) .
$$

We suppose throughout the paper that $f$ is transcendental; that is to say,

$$
\lim _{r \rightarrow \infty} \frac{T(r, f)}{\log r}=\infty
$$

and that $f$ is linearly non-degenerate over $C$; namely, $f_{1}, \ldots, f_{n+1}$ are linearly independent over $\boldsymbol{C}$.

Received February 5, 2004; revised October 14, 2004. 
It is well-known that $f$ is linearly non-degenerate over $\boldsymbol{C}$ if and only if the Wronskian $W=W\left(f_{1}, \ldots, f_{n+1}\right)$ of $f_{1}, \ldots, f_{n+1}$ is not identically equal to zero.

For meromorphic functions in the complex plane we use the standard notations of Nevanlinna theory of meromorphic functions ([4], [5]).

For $\boldsymbol{a} \in \boldsymbol{C}^{n+1}-\{\boldsymbol{0}\}$, we write

$$
\begin{aligned}
& m(r, \boldsymbol{a}, f)=\frac{1}{2 \pi} \int_{0}^{2 \pi} \log \frac{\|\boldsymbol{a}\|\left\|f\left(r e^{i \theta}\right)\right\|}{\left|\left(\boldsymbol{a}, f\left(r e^{i \theta}\right)\right)\right|} d \theta, \\
& N(r, \boldsymbol{a}, f)=N\left(r, \frac{1}{(\boldsymbol{a}, f)}\right) .
\end{aligned}
$$

We then have the first fundamental theorem:

$$
T(r, f)=m(r, \boldsymbol{a}, f)+N(r, \boldsymbol{a}, f)+O(1)
$$

([11], p. 76). We call the quantity

$$
\delta(\boldsymbol{a}, f)=1-\limsup _{r \rightarrow \infty} \frac{N(r, \boldsymbol{a}, f)}{T(r, f)}=\liminf _{r \rightarrow \infty} \frac{m(r, \boldsymbol{a}, f)}{T(r, f)}
$$

the deficiency (or defect) of $\boldsymbol{a}$ with respect to $f$. We have

$$
0 \leq \delta(\boldsymbol{a}, f) \leq 1
$$

by (2) since $N(r, \boldsymbol{a}, f) \geq 0$ for $r \geq 1$ and $m(r, \boldsymbol{a}, f) \geq 0$ for $r>0$.

Let $X$ be a subset of $\boldsymbol{C}^{n+1}-\{\boldsymbol{0}\}$ in $N$-subgeneral position; that is to say, $\# X \geq N+1$ and any $N+1$ elements of $X$ generate $C^{n+1}$, where $N$ is an integer satisfying $N \geq n$.

Cartan $([1], N=n)$ and Nochka $([6], N>n)$ gave the following

Theorem A (Defect relation). For any $q$ elements $\boldsymbol{a}_{j}(j=1, \ldots, q)$ of $X$,

$$
\sum_{j=1}^{q} \delta\left(\boldsymbol{a}_{j}, f\right) \leq 2 N-n+1,
$$

where $2 N-n+1 \leq q \leq \infty$ (see also [2] or [3]).

We are interested in the holomorphic curve $f$ for which the defect relation is extremal:

$$
\sum_{j=1}^{q} \delta\left(\boldsymbol{a}_{j}, f\right)=2 N-n+1
$$

In [9] we proved the following theorem.

THEOREM B. Suppose that there are vectors $\boldsymbol{a}_{j}(j=1, \ldots, q)$ in $X$ which satisfy (3), where $2 N-n+1 \leq q \leq \infty$. If $(n+1,2 N-n+1)=1$, then there are at least 


$$
\left[\frac{2 N-n+1}{n+1}\right]+1
$$

vectors $\boldsymbol{a} \in\left\{\boldsymbol{a}_{j}(j=1, \ldots, q)\right\}$ satisfying $\delta(\boldsymbol{a}, f)=1$.

Further, we improved this theorem in [10]. Namely, we weakened the condition " $(n+1,2 N-n+1)=1$ " in Theorem B to " $N>n=2 m(m \in N)$ " and obtained the same conclusion as in Theorem B.

In this paper we consider the holomorphic curve $f$ satisfying (3) from a different point of view.

Let

$$
X_{k}(0)=\left\{\boldsymbol{a}=\left(a_{1}, \ldots, a_{n+1}\right) \in X \mid a_{k}=0\right\} \quad(1 \leq k \leq n+1) .
$$

Then, it is easy to see that

$$
0 \leq \# X_{k}(0) \leq N
$$

since $X$ is in $N$-subgeneral position.

Further we put (see Definition 1 in [7])

$$
\begin{gathered}
u_{k}(z)=\max _{1 \leq j \leq n+1, j \neq k}\left|f_{j}(z)\right|, \\
t_{k}(r, f)=\frac{1}{2 \pi} \int_{0}^{2 \pi}\left\{\log u_{k}\left(r e^{i \theta}\right)-\log u_{k}\left(e^{i \theta}\right)\right\} d \theta,
\end{gathered}
$$

and

$$
\Omega_{k}=\limsup _{r \rightarrow \infty} \frac{t_{k}(r, f)}{T(r, f)}
$$

Proposition A (see [7]).

(a) $t_{k}(r, f)$ is independent of the choice of reduced representation of $f$.

(b) $t_{k}(r, f) \leq T(r, f)+O(1)$.

(c) $N\left(r, 1 / f_{j}\right) \leq t_{k}(r, f)+O(1)(j=1, \ldots, n+1, j \neq k)$.

(d) $0 \leq \Omega_{k} \leq 1$.

Our main purpose of this paper is to prove the following theorem:

THEOREM. Suppose that

(i) $N>n \geq 2$;

(ii) there are vectors $\boldsymbol{a}_{1}, \ldots, \boldsymbol{a}_{q} \in X \quad(2 N-n+1<q \leq \infty)$ satisfying $\delta\left(\boldsymbol{a}_{j}, f\right)>0(j=1, \ldots, q)$ and

$$
\sum_{j=1}^{q} \delta\left(\boldsymbol{a}_{j}, f\right)=2 N-n+1 .
$$

If $\Omega_{k}<1$ for some $k(1 \leq k \leq n+1)$, then 
(a) $\# X_{k}(0)=N$;

(b) there is a subset $P \subset\{1,2, \ldots, q\}$ satisfying

$$
\# P=N-n+1, \quad d(P)=1, \quad \delta\left(\boldsymbol{a}_{j}, f\right)=1 \quad(j \in P)
$$

and

$$
X_{k}(0) \cap\left\{\boldsymbol{a}_{j} \mid j \in P\right\}=\phi,
$$

where $d(P)$ is the dimension of the vector space spanned by $\left\{\boldsymbol{a}_{j} \mid j \in P\right\}$.

(c) Any $n$ elements of $X-\left\{\boldsymbol{a}_{j} \mid j \in P\right\}$ are linearly independent.

As an application of this theorem, we can prove the following result:

"Let $f$ be any exponential curve. If $N>n \geq 2$, then

$$
\sum_{\boldsymbol{a} \in X} \delta(\boldsymbol{a}, f)<2 N-n+1 . "
$$

This result means that any exponential curve is not extremal for the defect relation when $N>n \geq 2$.

\section{Preliminaries and lemma}

We shall give some lemmas for later use. Let $f=\left[f_{1}, \ldots, f_{n+1}\right], X$ and $X_{k}(0)$ etc. be as in Section $1, q$ any integer satisfying $2 N-n+1<q<\infty$ and we put $Q=\{1,2, \ldots, q\}$.

Let $\left\{\boldsymbol{a}_{j} \mid j \in Q\right\}$ be a family of vectors in $X$. For a non-empty subset $P$ of $Q$, we denote

$V(P)=$ the vector space spanned by $\left\{\boldsymbol{a}_{j} \mid j \in P\right\} \quad$ and $\quad d(P)=\operatorname{dim} V(P)$ and we put

$$
\mathcal{O}=\{P \subset Q \mid 0<\# P \leq N+1\}
$$

Lemma $1((2.4 .3)$ in [3], p. 68). For $P \in \mathcal{O}, \# P-d(P) \leq N-n$.

For $\left\{\boldsymbol{a}_{j} \mid j \in Q\right\}$, let

$$
\omega: Q \rightarrow(0,1]
$$

be the Nochka weight function given in [3, p. 72] and $\theta$ the reciprocal number of the Nochka constant given in $[3, \mathrm{p}$. 72]. Then they have the following properties:

LEMMA 2 (see [3], Theorem 2.11.4).

(a) $0<\omega(j) \theta \leq 1$ for all $j \in Q$;

(b) $q-2 N+n-1=\theta\left(\sum_{j=1}^{q} \omega(j)-n-1\right)$;

(c) $(N+1) /(n+1) \leq \theta \leq(2 N-n+1) /(n+1)$;

(d) If $P \in \mathcal{O}$, then $\sum_{j \in P} \omega(j) \leq d(P)$. 
Note 1. (c) of Lemma 2 can be refined as follows: $\frac{N}{n} \leq \theta \leq \frac{2 N-n+1}{n+1}$.

Proof. When $\theta=(2 N-n+1) /(n+1)$, there is nothing to prove as $N / n \leq$ $(2 N-n+1) /(n+1)$.

When $\theta<(2 N-n+1) /(n+1)$, there is an element $P \in \mathcal{O}$ satisfying

$$
\theta=\frac{2 N-n+1-\# P}{n+1-d(P)} \quad(1 \leq d(P) \leq n)
$$

by the definition of $\theta$. By Lemma 1 we have

$$
\theta=\frac{2 N-n+1-\# P}{n+1-d(P)} \geq \frac{N+1-d(P)}{n+1-d(P)} \geq \frac{N}{n}
$$

since $d(P) \geq 1$.

Put

$$
P_{k}(0)=\left\{j \in Q \mid \boldsymbol{a}_{j} \in X_{k}(0)\right\} \quad \text { and } \quad d_{k}=\sum_{j \in P_{k}(0)} \omega(j) .
$$

Then, we have the inequality

$$
d_{k} \leq n
$$

since $d_{k} \leq d\left(P_{k}(0)\right)$ by Lemma $2(\mathrm{~d})$ and $d\left(P_{k}(0)\right) \leq n$ by the definition of $X_{k}(0)$.

Lemma 3 (Defect relation) (see Theorem 3 in [8]). For any $\boldsymbol{a}_{1}, \ldots, \boldsymbol{a}_{q} \in X$, we have the following inequalities:

(I) $\sum_{j=1}^{q} \omega(j) \delta\left(\boldsymbol{a}_{j}, f\right) \leq d_{k}+1+\left(n-d_{k}\right) \Omega_{k}$;

(II) $\sum_{j=1}^{q} \delta\left(\boldsymbol{a}_{j}, f\right) \leq 2 N-n+1-\frac{N}{n}\left(n-d_{k}\right)\left(1-\Omega_{k}\right)$.

By applying Lemma 2 and Note 1 to (I) we obtain (II) as usual. (II) is an amelioration of Theorem 3 (II) in [8].

Remark 1. This is an amelioration of Theorem A. Since $\Omega_{k} \leq 1$ and $d_{k} \leq n$ we have the inequalities:

$$
\begin{gathered}
d_{k}+1+\left(n-d_{k}\right) \Omega_{k} \leq n+1 \text { and } \\
2 N-n+1-N\left(n-d_{k}\right)\left(1-\Omega_{k}\right) / n \leq 2 N-n+1 .
\end{gathered}
$$

Lemma 4. For any $\boldsymbol{a} \in X_{k}(0), \delta(\boldsymbol{a}, f) \geq 1-\Omega_{k}$.

Proof. For $\boldsymbol{a} \in X_{k}(0)$ we have the inequality

$$
|(\boldsymbol{a}, f(z))| \leq K u_{k}(z)
$$


for a positive constant $K$ by the definitions of $X_{k}(0)$ and $u_{k}(z)$. From this inequality we have the inequality

$$
\begin{aligned}
N(r, \boldsymbol{a}, f)=\frac{1}{2 \pi} \int_{0}^{2 \pi} \log \left|\left(\boldsymbol{a}, f\left(r e^{i \theta}\right)\right)\right| d \theta & \leq \frac{1}{2 \pi} \int_{0}^{2 \pi} \log u_{k}\left(r e^{i \theta}\right) d \theta+\log K \\
& =t_{k}(r, f)+O(1) \quad(r>0),
\end{aligned}
$$

from which we obtain the inequality $\delta(\boldsymbol{a}, f) \geq 1-\Omega_{k}$.

Lemma 5 ([9], Lemma 3). Suppose that $N>n$. For $\boldsymbol{a}_{1}, \ldots, \boldsymbol{a}_{q} \in X$ the maximal deficiency sum

$$
\sum_{j=1}^{q} \delta\left(\boldsymbol{a}_{j}, f\right)=2 N-n+1
$$

holds if and only if the following two relations hold:

1) $(1-\theta \omega(j))\left(1-\delta\left(\boldsymbol{a}_{j}, f\right)\right)=0(j=1, \ldots, q)$;

2) $\sum_{j=1}^{q} \omega(j) \delta\left(\boldsymbol{a}_{j}, f\right)=n+1$.

Corollary 1 ([9], Corollary 1(I)). Suppose that $N>n$ and that for $\boldsymbol{a}_{1}, \ldots, \boldsymbol{a}_{q} \in X$, the equality

$$
\sum_{j=1}^{q} \delta\left(\boldsymbol{a}_{j}, f\right)=2 N-n+1
$$

holds. If $\theta \omega(j)<1$ for some $j$, then $\delta\left(\boldsymbol{a}_{j}, f\right)=1$.

Definition 1 ([9], Definition 1). We put

$$
\lambda=\min _{P \in \mathcal{O}} \frac{d(P)}{\# P} .
$$

Then, $\lambda$ has the following property.

Lemma 6 ([9], Proposition 2). $1 /(N-n+1) \leq \lambda \leq(n+1) /(N+1)$.

Remark 2. (a) If $\lambda<(n+1) /(2 N-n+1)$, then $\lambda=\min _{1 \leq j \leq q} \omega(j)$ and $\omega(j)=\lambda, \theta \omega(j)<1\left(j \in P_{0}\right)$ for an element $P_{0} \in \mathcal{O}$ satisfying $\lambda=d\left(P_{0}\right) / \# P_{0}$.

(b) If $\lambda \geq(n+1) /(2 N-n+1)$, then $\omega(j)=1 / \theta=(n+1) /(2 N-n+1)$ $(j=1, \ldots, q)$.

In fact, the first assertion of (a) is given in the proof of Proposition 2.4.4 ([3], p. 68) with the definition of $\omega(j)([3]$, p. 72). For the second assertion of (a), as $\omega(j)=\lambda\left(j \in P_{0}\right)$ by the definition of $\omega$ and $(n+1) /(2 N-n+1) \leq 1 / \theta$, we have the conclusion.

(b) See the definitions of $\omega(j)$ and $\theta([3]$, p. 72). 
Lemma 7 ([9], Corollary 2). For $\boldsymbol{a}_{1}, \ldots, \boldsymbol{a}_{q} \in X$, we have the inequality

$$
\sum_{j=1}^{q} \delta\left(\boldsymbol{a}_{j}, f\right) \leq \min \left(2 N-n+1, \frac{n+1}{\lambda}\right) .
$$

\section{Proof of Theorem when $q<\infty$}

Let $f, X, X_{k}(0)$ and $\omega$ etc. be as in the previous sections and $q$ an integer satisfying

$$
2 N-n+1<q<\infty .
$$

Throughout this section we suppose that

(i) $N>n \geq 2$;

(ii) there are vectors $\boldsymbol{a}_{1}, \ldots, \boldsymbol{a}_{q} \in X$ satisfying $\delta\left(\boldsymbol{a}_{j}, f\right)>0(j=1, \ldots, q)$ and

$$
\sum_{j=1}^{q} \delta\left(\boldsymbol{a}_{j}, f\right)=2 N-n+1
$$

(iii) $\Omega_{k}<1$ for some $k(1 \leq k \leq n+1)$.

Proposition 1. $X_{k}(0) \subset\left\{\boldsymbol{a}_{1}, \ldots, \boldsymbol{a}_{q}\right\}$.

Proof. If there exists a vector $\boldsymbol{a} \in X_{k}(0)$ satisfying $\boldsymbol{a} \notin\left\{\boldsymbol{a}_{1}, \ldots, \boldsymbol{a}_{q}\right\}$, then $\delta(\boldsymbol{a}, f)>0$ by Lemma 4 and (iii), and so by Theorem $\mathrm{A}$ we have the inequality

$$
\sum_{j=1}^{q} \delta\left(\boldsymbol{a}_{j}, f\right) \leq 2 N-n+1-\delta(\boldsymbol{a}, f)<2 N-n+1,
$$

which is a contradiction to our assumption (ii).

PROPOSITION 2. $d_{k}=n$.

Proof. From Lemma 3(II) and the assumption (ii) we have the inequality

$$
\left(1-\Omega_{k}\right)\left(n-d_{k}\right) \leq 0 .
$$

Then, by the assumption (iii) and (4), we obtain the equality $d_{k}=n$.

Proposition 3. (a) $\theta=N / n$, (b) $\# P_{k}(0)=N$ and (c) $\theta \omega(j)=1\left(j \in P_{k}(0)\right)$.

Proof. As $X$ is in $N$-subgeneral position, we have $\# X_{k}(0) \leq N$. From Proposition 2 and Lemma 2(a)

$$
\text { (A) } \theta n=\sum_{j \in P_{k}(0)} \theta \omega(j) \leq \sum_{j \in P_{k}(0)} 1=\# P_{k}(0)=\# X_{k}(0) \leq N,
$$

so that we have $\theta \leq N / n$. By Note 1 we obtain $\theta=N / n$. 
Combining this result with the inequality (A), we have

$$
\# P_{k}(0)=N \quad \text { and } \quad \theta \omega(j)=1 \quad\left(j \in P_{k}(0)\right) .
$$

Corollary 2. $\lambda<(n+1) /(2 N-n+1)$.

Proof. By Lemma 7 and the assumption (ii), we have

$$
\lambda \leq \frac{n+1}{2 N-n+1} .
$$

If $\lambda=(n+1) /(2 N-n+1)$, then by Remark 2(b) and Proposition 3(a)

$$
\theta=\frac{2 N-n+1}{n+1}=\frac{N}{n},
$$

which is a contradiction, since $N / n<(2 N-n+1) /(n+1)$ when $N>n \geq 2$. This implies that our corollary holds.

Put

$$
P_{1}=\{j \mid \theta \omega(j)<1,1 \leq j \leq q\}
$$

Then,

$$
P_{1} \cap P_{k}(0)=\phi
$$

by Proposition 3(c). We have the following

Proposition 4. $N-n+1 \leq \# P_{1}<2 N-n+1$.

Proof. (a) From Lemma 2(b), we have

$$
q-(2 N-n+1)=\theta\left(\sum_{j=1}^{q} \omega(j)-n-1\right)=\sum_{j \notin P_{1}} \theta \omega(j)+\sum_{j \in P_{1}} \theta \omega(j)-\theta n-\theta .
$$

Here, as $\theta \omega(j)=1$ for $j \notin P_{1}$ we have that

$$
\sum_{j \notin P_{1}} \theta \omega(j)=q-\# P_{1}
$$

and by Proposition 3(a) we have

$$
\sum_{j \in P_{1}} \theta \omega(j)-\theta n-\theta=-N+\frac{N}{n}\left(\sum_{j \in P_{1}} \omega(j)-1\right) .
$$

Combining these three equalities we obtain

$$
\text { (B) } \quad q-(2 N-n+1)=q-\# P_{1}-N+\frac{N}{n}\left(\sum_{j \in P_{1}} \omega(j)-1\right) .
$$


Here, as $1 /(N-n+1) \leq \lambda \leq \omega(j)\left(j \in P_{1}\right)$ due to Lemma 6, Corollary 2 and Remark 2(a) we obtain the inequality

$$
\left(\mathrm{B}_{2}\right) \quad q-\# P_{1}-N+\frac{N}{n}\left(\sum_{j \in P_{1}} \omega(j)-1\right) \geq q-\# P_{1}-N+\frac{N}{n}\left(\frac{\# P_{1}}{N-n+1}-1\right) \text {. }
$$

From $\left(B_{1}\right)$ and $\left(B_{2}\right)$ we have the inequality

$$
q-(2 N-n+1) \geq q-\# P_{1}-N+\frac{N}{n}\left(\frac{\# P_{1}}{N-n+1}-1\right),
$$

which reduces to the inequality

$$
(N-n+1-N / n)\left(\# P_{1}-N+n-1\right) \geq 0 .
$$

As

$$
N-n+1-\frac{N}{n}=\frac{(N-n)(n-1)}{n}>0
$$

by the assumption (i), we have

$$
\# P_{1} \geq N-n+1 \text {. }
$$

(b) As $\delta\left(\boldsymbol{a}_{j}, f\right)=1\left(j \in P_{1}\right)$ by Corollary 1, from Propositions 1, 3(b) and the assumption (ii) we have

$$
\# P_{1}<2 N-n+1
$$

as $P_{1} \cap P_{k}(0)=\phi$ by $(5)$.

Let $P_{0}$ be an element of $\mathcal{O}$ satisfying

$$
\frac{d\left(P_{0}\right)}{\# P_{0}}=\lambda,
$$

where $\lambda=\min _{P \in \mathcal{O}} d(P) / \# P$. Then, $\omega(j)=\lambda\left(j \in P_{0}\right)$ and

$$
\phi \neq P_{0} \subset P_{1}
$$

since $\theta \lambda<1$ by Corollary 2 and Remark 2(a).

Proposition 5. (a) $\# P_{0}=N-n+1$, (b) $d\left(P_{0}\right)=1$ and

(c) $\omega(j)=\lambda=1 /(N-n+1)\left(j \in P_{0}\right)$.

Proof. By Proposition 3(a), $\theta$ is equal to $N / n$, which is smaller than $(2 N-n+1) /(n+1)$. By the definition of $\theta$, there exists a set $P \in \mathcal{O}$ satisfying

$$
P_{0} \subset P, \quad 1 \leq d(P) \leq n
$$

and

$$
\theta=\frac{2 N-n+1-\# P}{n+1-d(P)}=\frac{N}{n}
$$


By Proposition 3(a) and Lemma 1 we have the inequality

$$
\begin{aligned}
0 & =\theta-\frac{N}{n}=\frac{2 N-n+1-\# P}{n+1-d(P)}-\frac{N}{n}=\frac{(N-n)(n-1)+N d(P)-n \# P}{n(n+1-d(P))} \\
& \geq \frac{(N-n)(d(P)-1)}{n(n+1-d(P))} \geq 0,
\end{aligned}
$$

which implies that

$$
d(P)=1 \quad \text { and } \quad \# P=N-n+1 .
$$

By Lemma 2(d), Remark 2(a) with Corollary 2 and Lemma 6 we obtain the inequality

$$
1=d(P) \geq \sum_{j \in P} \omega(j) \geq(N-n+1) \lambda \geq 1
$$

and we have

$$
\lambda=\frac{1}{N-n+1}=\omega(j) \quad(j \in P) .
$$

By the choice of $P_{0}, 1 \leq d\left(P_{0}\right) \leq d(P)=1$ and so we have

$$
d\left(P_{0}\right)=1 \text { and } \# P_{0}=N-n+1 .
$$

Proposition 6. $P_{1}=P_{0}$.

Proof. By Lemma 2(b) we have the equality

$$
\begin{aligned}
q-(2 N-n+1) & =\theta\left(\sum_{j=1}^{q} \omega(j)-n-1\right) \\
& =\theta\left(\sum_{j \notin P_{k}(0) \cup P_{0}} \omega(j)+\sum_{j \in P_{k}(0) \cup P_{0}} \omega(j)-n-1\right) .
\end{aligned}
$$

Here, as $P_{k}(0) \cap P_{0}=\phi$ by (5) and (6), $\sum_{j \in P_{k}(0)} \omega(j)=d_{k}=n$ (Proposition 2) and $\sum_{j \in P_{0}} \omega(j)=1$ (Proposition 5(a), (c)), we have

$$
\sum_{j \in P_{k}(0) \cup P_{0}} \omega(j)=\sum_{j \in P_{k}(0)} \omega(j)+\sum_{j \in P_{0}} \omega(j)=n+1,
$$

so that we have the equality

$$
\left(\mathrm{C}_{1}\right) \quad q-(2 N-n+1)=\theta \sum_{j \notin P_{k}(0) \cup P_{0}} \omega(j) .
$$

As $P_{0} \subset P_{1}, \theta \omega(j)=1$ for $j \notin P_{1}$ and $\theta \omega(j)<1$ for $j \in P_{1}$ by the definition 
of $P_{1}, \# P_{k}(0)=N$ (Proposition 3(b)), $\# P_{0}=N-n+1$ (Proposition 5(a)) and $P_{k}(0) \cap P_{1}=\phi$ by (5), we have

$$
\begin{aligned}
\left(\mathrm{C}_{2}\right) \quad \theta \sum_{j \notin P_{k}(0) \cup P_{0}} \omega(j) & =q-(2 N-n+1)-\#\left(P_{1}-P_{0}\right)+\theta \sum_{j \in P_{1}-P_{0}} \omega(j) \\
& =q-(2 N-n+1)-\sum_{j \in P_{1}-P_{0}}(1-\theta \omega(j)),
\end{aligned}
$$

From $\left(\mathrm{C}_{1}\right)$ and $\left(\mathrm{C}_{2}\right)$ we have the equality

$$
\sum_{j \in P_{1}-P_{0}}(1-\theta \omega(j))=0 .
$$

If $P_{0} \varsubsetneqq P_{1}$, we have a contradiction since $1-\theta \omega(j)>0$ for $j \in P_{1}$. This means that $P_{1}=P_{0}$ must hold.

Proposition 7. Any $n$ elements of $X-\left\{\boldsymbol{a}_{j} \mid j \in P_{0}\right\}$ are linearly independent. In particular, any $n$ elements of $X_{k}(0)$ are linearly independent.

Proof. Let $\boldsymbol{b}_{1}, \ldots, \boldsymbol{b}_{n}$ be any $n$ elements of $X-\left\{\boldsymbol{a}_{j} \mid j \in P_{0}\right\}$. Then, the set

$$
\left\{\boldsymbol{b}_{1}, \ldots, \boldsymbol{b}_{n}\right\} \cup\left\{\boldsymbol{a}_{j} \mid j \in P_{0}\right\}
$$

contains $n+1$ linearly independent elements since $X$ is in $N$-subgeneral position. As $d\left(P_{0}\right)=1, \boldsymbol{b}_{1}, \ldots, \boldsymbol{b}_{n}$ must be linearly independent. As $X_{k}(0) \subset X-$ $\left\{\boldsymbol{a}_{j} \mid j \in P_{0}\right\}$, we have the last assertion.

Summarizing Propositions from 1 through 7, we obtain the following

THEOREM 1. Suppose that

(i) $N>n \geq 2$;

(ii) there are vectors $\boldsymbol{a}_{1}, \ldots, \boldsymbol{a}_{q} \in X(2 N-n+1<q<\infty)$ satisfying

$$
\sum_{j=1}^{q} \delta\left(\boldsymbol{a}_{j}, f\right)=2 N-n+1 .
$$

If $\Omega_{k}<1$ for some $k(1 \leq k \leq n+1)$, then

(a) $X_{k}(0) \subset\left\{\boldsymbol{a}_{1}, \ldots, \boldsymbol{a}_{q}\right\}$ and $\# X_{k}(0)=N$;

(b) there is a subset $P \subset Q$ satisfying

$$
\# P=N-n+1, \quad d(P)=1, \quad \delta\left(\boldsymbol{a}_{j}, f\right)=1 \quad(j \in P)
$$

and

$$
X_{k}(0) \cap\left\{\boldsymbol{a}_{j} \mid j \in P\right\}=\phi ;
$$

(c) any $n$ elements of $X-\left\{\boldsymbol{a}_{j} \mid j \in P\right\}$ are linearly independent. 


\section{Proof of Theorem when $q=\infty$}

Let $\left[f_{1}, \ldots, f_{n+1}\right], X, X_{k}(0), \theta$ and $\omega$ etc. be as in Section 1,2 or 3 . From Theorem A, it is easy to see that the set

$$
\{\boldsymbol{a} \in X \mid \delta(\boldsymbol{a}, f)>0\}
$$

is at most countable and

$$
\sum_{\boldsymbol{a} \in X} \delta(\boldsymbol{a}, f) \leq 2 N-n+1 .
$$

In this section we consider a holomorphic curve $f$ with an infinite number of vectors $\boldsymbol{a}_{j} \in X$ such that

$$
\delta\left(\boldsymbol{a}_{j}, f\right)>0 \quad(j=1,2,3, \ldots) .
$$

We put

$$
\begin{aligned}
\boldsymbol{N} & =\{1,2,3, \ldots\} \quad \text { (the set of positive integers); } \\
Y & =\left\{\boldsymbol{a}_{j} \mid j \in \boldsymbol{N}\right\} ; \\
\mathcal{O}_{\infty} & =\{P \subset \boldsymbol{N} \mid 0<\# P \leq N+1\}
\end{aligned}
$$

and for any non-empty, finite subset $P$ of $N$, we use

$$
V(P) \text { and } d(P)
$$

as in Section 2.

Definition 2 (see [9], p. 144). We put

$$
\lambda_{\infty}=\min _{P \in \mathcal{O}_{\infty}} \frac{d(P)}{\# P} .
$$

Note that the set $\left\{d(P) / \# P \mid P \in \mathcal{O}_{\infty}\right\}$ is a finite set. We have the followings ([9], p. 144):

$\left(a_{\infty}\right) 1 /(N-n+1) \leq \lambda_{\infty} \leq(n+1) /(N+1)$;

$\left(b_{\infty}\right)$ (the inequality $(12)$ in [9])

$$
\sum_{j=1}^{\infty} \delta\left(\boldsymbol{a}_{j}, f\right) \leq(n+1) / \lambda_{\infty} .
$$

From now on throughout this section we suppose that

(i) $N>n \geq 2$;

(ii) there exists a subset $Y=\left\{\boldsymbol{a}_{j} \mid j \in \boldsymbol{N}\right\}$ of $X$ satisfying $\delta\left(\boldsymbol{a}_{j}, f\right)>0$ and

$$
\sum_{j=1}^{\infty} \delta\left(\boldsymbol{a}_{j}, f\right)=2 N-n+1
$$

(iii) $\Omega_{k}<1$ for some $k(1 \leq k \leq n+1)$. 
Note that we obtain the inequality

$$
\lambda_{\infty} \leq(n+1) /(2 N-n+1)
$$

from (7) and $\left(b_{\infty}\right)$. Let $P_{0}$ be an element of $\mathcal{O}_{\infty}$ satisfying

$$
\frac{d\left(P_{0}\right)}{\# P_{0}}=\lambda_{\infty}
$$

and let $\varepsilon$ be any positive number satisfying

$$
0<\varepsilon<(N-n)\left(1-\Omega_{k}\right) /(N-n+1)(n+1) .
$$

We restrict the number $\varepsilon$ as in (9) for the forthcoming Propositions from 8 through 13 to hold.

Now, for the number $\varepsilon$ in (9), there exists $p \in N$ satisfying $\{1,2, \ldots, p\} \supset P_{0}$, $p>2 N-n+1$ and

$$
2 N-n+1-\varepsilon<\sum_{j=1}^{p} \delta\left(\boldsymbol{a}_{j}, f\right) .
$$

For an integer $q$ not less than $p$, we put

$$
Q=\{1,2, \ldots, q\} .
$$

Note that $2 N-n+1<q<\infty$. For this $Q$, we use $\theta_{q}, \omega_{q}$ and $\lambda_{q}$ instead of $\theta$, $\omega$ and $\lambda$ in Section 2 respectively. Note that

$$
\lambda_{q}=\lambda_{\infty}
$$

since $Q \supset P_{0}$. Further we obtain the following inequalities from the equality (2) in $[9]$ :

$$
\begin{gathered}
n+1-\frac{\varepsilon}{\theta_{q}}<\sum_{j=1}^{q} \omega_{q}(j) \delta\left(\boldsymbol{a}_{j}, f\right) \\
\sum_{j=1}^{q}\left(1-\theta_{q} \omega_{q}(j)\right)\left(1-\delta\left(\boldsymbol{a}_{j}, f\right)\right)<\varepsilon .
\end{gathered}
$$

From now on we put $\varepsilon_{1}=\varepsilon /\left(1-\Omega_{k}\right)$ for simplicity. Then,

$$
0<\varepsilon_{1}<(N-n) /(N-n+1)(n+1) .
$$

Proposition 8. $X_{k}(0) \subset\left\{\boldsymbol{a}_{1}, \ldots, \boldsymbol{a}_{q}\right\}$.

Proof. If there exists a vector $\boldsymbol{a} \in X_{k}(0)$ satisfying $\boldsymbol{a} \notin\left\{\boldsymbol{a}_{1}, \ldots, \boldsymbol{a}_{q}\right\}$, then by Lemma 4, Theorem A and (10) we have the inequality 


$$
\begin{aligned}
2 N-n+1-\varepsilon & <\sum_{j=1}^{q} \delta\left(\boldsymbol{a}_{j}, f\right) \leq 2 N-n+1-\delta(\boldsymbol{a}, f) \leq 2 N-n+1-\left(1-\Omega_{k}\right) \\
& <2 N-n+1-\varepsilon
\end{aligned}
$$

as $p \leq q$ and $\varepsilon<1-\Omega_{k}$ from (9). This is a contradiction.

We put

$$
P_{k}(0)=\left\{j \in Q \mid \boldsymbol{a}_{j} \in X_{k}(0)\right\} \quad \text { and } \quad d_{k}(q)=\sum_{j \in P_{k}(0)} \omega_{q}(j) .
$$

Note that

$$
\# P_{k}(0) \leq N \quad \text { and } \quad d_{k}(q) \leq d\left(P_{k}(0)\right) \leq n .
$$

In fact, we have $\# P_{k}(0)=\# X_{k}(0) \leq N$ as $X$ is in $N$-subgeneral position. We have $d_{k}(q) \leq d\left(P_{k}(0)\right)$ by Lemma $2(\mathrm{~d})$ and $d\left(P_{k}(0)\right) \leq n$ by the definition of $X_{k}(0)$.

PROPOSITION 9. $n-\varepsilon_{1} / \theta_{q}<d_{k}(q)$.

Proof. From (12) and Lemma 3(I) we have the inequality

$$
n+1-\varepsilon / \theta_{q}<\sum_{j=1}^{q} \omega_{q}(j) \delta\left(\boldsymbol{a}_{j}, f\right) \leq d_{k}(q)+1+\left(n-d_{k}(q)\right) \Omega_{k}
$$

from which we obtain

$$
\left(n-d_{k}(q)\right)\left(1-\Omega_{k}\right)<\varepsilon / \theta_{q}
$$

and so $n-\varepsilon_{1} / \theta_{q}<d_{k}(q)$ as $\Omega_{k}<1$ and $\varepsilon_{1}=\varepsilon /\left(1-\Omega_{k}\right)$.

Proposition 10. (a) $\theta_{q}<\left(N+\varepsilon_{1}\right) / n$ and (b) $\# P_{k}(0)=N$.

Proof. From Proposition 9, Lemma 2(a) and (15), we have the inequality

$$
\text { (D) } \theta_{q}\left(n-\varepsilon_{1} / \theta_{q}\right)<\theta_{q} d_{k}(q)=\theta_{q} \sum_{j \in P_{k}(0)} \omega_{q}(j) \leq \# P_{k}(0) \leq N \text {, }
$$

from which we obtain $\theta_{q}<\left(N+\varepsilon_{1}\right) / n$ easily. Next, from (D) and Note 1, we obtain that $N-\varepsilon_{1}<\# P_{k}(0) \leq N$, so that $\# P_{k}(0)=N$ as $\varepsilon_{1}<1$ from (14).

Corollary 3. (a) $\theta_{q} \lambda_{q}<1$ and (b) $\lambda_{q}<(n+1) /(2 N-n+1)$.

Proof. (a) From (8), (11) and Proposition 10(a), we have 
ON A CERTAIN HOLOMORPHIC CURVE EXTREMAL FOR THE DEFECT RELATION

$$
\theta_{q} \lambda_{q}<\frac{N+\varepsilon_{1}}{n} \frac{n+1}{2 N-n+1}
$$

and by (14) and the assumption (i) it is easy to see that

$$
\frac{N+\varepsilon_{1}}{n} \frac{n+1}{2 N-n+1}<1 \text {. }
$$

We have (a) of this corollary.

(b) By (8) and (11), we have $\lambda_{q} \leq(n+1) /(2 N-n+1)$. If $\lambda_{q}$ is equal to $(n+1) /(2 N-n+1)$, then by Remark $2(\mathrm{~b})$ we have $\theta_{q} \lambda_{q}=1$, which contradicts (a) of this corollary. We have (b) of this corollary.

Put

$$
P_{1}=\left\{j \in Q \mid \theta_{q} \omega_{q}(j)<1, j \notin P_{k}(0)\right\} .
$$

Note that

$$
P_{1} \cap P_{k}(0)=\phi
$$

Proposition 11. $N-n+1 \leq \# P_{1}$.

Proof. From Lemma 2(b) and (16) we have the equality

( $\left.\mathrm{E}_{1}\right) \quad q-(2 N-n+1)=\theta_{q}\left\{\sum_{j=1}^{q} \omega_{q}(j)-n-1\right\}$

$$
=\theta_{q}\left\{\sum_{j \in P_{k}(0)} \omega_{q}(j)+\sum_{j \in P_{1}} \omega_{q}(j)+\sum_{j \notin P_{k}(0) \cup P_{1}} \omega_{q}(j)-n-1\right\}
$$

and by Proposition 9

$$
>\theta_{q}\left\{\sum_{j \in P_{1}} \omega_{q}(j)+\sum_{j \notin P_{k}(0) \cup P_{1}} \omega_{q}(j)-\left(1+\varepsilon_{1} / \theta_{q}\right)\right\} .
$$

Here, by $\left(a_{\infty}\right),(11)$ and Remark 2(a) with Corollary 3(b) we have

$$
\sum_{j \in P_{1}} \omega_{q}(j) \geq \frac{\# P_{1}}{N-n+1}
$$

and as $\theta_{q} \omega_{q}(j)=1$ for $j \notin P_{k}(0) \cup P_{1}$ by Lemma $2\left(\right.$ a) and the definition of $P_{1}$, we have

$$
\theta_{q} \sum_{j \notin P_{k}(0) \cup P_{1}} \omega_{q}(j)=q-\# P_{k}(0)-\# P_{1},
$$


so that we have the inequality

$\left(\mathrm{E}_{2}\right)$ the last term of $\left(\mathrm{E}_{1}\right) \geq \frac{\theta_{q} \# P_{1}}{(N-n+1)}+q-\# P_{k}(0)-\# P_{1}-\theta_{q}-\varepsilon_{1}$.

From $\left(E_{1}\right)$ and $\left(E_{2}\right)$ we obtain the following inequality by Proposition $10(b)$

$$
\# P_{1}\left(1-\frac{\theta_{q}}{N-n+1}\right)>N-n+1-\theta_{q}-\varepsilon_{1},
$$

which reduces to the inequality

$$
\# P_{1}\left(N-n+1-\theta_{q}\right)>(N-n+1)\left(N-n+1-\theta_{q}-\varepsilon_{1}\right) .
$$

Here, by Proposition 10 (a) and by the fact that $0<\varepsilon_{1}<1$ from (14) we have the inequality

$$
N-n+1-\theta_{q}>N-n+1-\frac{N+\varepsilon_{1}}{n}=\frac{(N-n)(n-1)-\varepsilon_{1}}{n}>0
$$

as $N>n \geq 2$ (the assumption (ii)), so that we have

$$
\begin{aligned}
\# P_{1} & >(N-n+1)\left(1-\frac{\varepsilon_{1}}{N-n+1-\theta_{q}}\right) \\
& >(N-n+1)\left(1-\frac{\varepsilon_{1}}{(N-n+1-(2 N-n+1) /(n+1))}\right) \\
& =(N-n+1)\left(1-\frac{(n+1) \varepsilon_{1}}{(N-n)(n-1)}\right) \\
& >N-n
\end{aligned}
$$

by Lemma $2(\mathrm{c})$ and (14). This means that $\# P_{1} \geq N-n+1$.

Proposition 12. (a) $\# P_{0}=N-n+1$, (b) $d\left(P_{0}\right)=1$ and (c) $\theta_{q}=N / n$.

Proof. By the definition of $\theta_{q}$ and the choice of $P_{0}$, there exists a set $P$ satisfying

$$
P_{0} \subset P, \quad 1 \leq d(P) \leq n
$$

and

$$
\theta_{q}=\frac{2 N-n+1-\# P}{n+1-d(P)} .
$$

By Proposition 10(a), (17) and Lemma 1 we have the inequality 


$$
\text { (F) } \quad \begin{aligned}
0 & >\theta_{q}-\left(N+\varepsilon_{1}\right) / n=\theta_{q}-N / n-\varepsilon_{1} / n \\
& =\frac{(N-n)(n-1)+N d(P)-n \# P}{n(n+1-d(P))}-\frac{\varepsilon_{1}}{n} \\
& \geq \frac{(N-n)(d(P)-1)}{n(n+1-d(P))}-\frac{\varepsilon_{1}}{n} .
\end{aligned}
$$

First we prove that $d(P)=1$. Suppose that $d(P) \geq 2$. Then, from $(\mathrm{F})$ we have the inequality

$$
\frac{\varepsilon_{1}}{n}>\frac{N-n}{n(n-1)},
$$

which reduces to the inequality

$$
\varepsilon_{1}>(N-n) /(n-1),
$$

which contradicts (14). This means that $d(P)$ must be equal to 1 .

As $d(P)=1$, we have from (17) and Note 1 that

$$
\theta_{q}=\frac{2 N-n+1-\# P}{n} \geq \frac{N}{n}
$$

from which we have that $\# P \leq N-n+1$. On the other hand, as

$$
\theta_{q}=\frac{2 N-n+1-\# P}{n}<\frac{N+\varepsilon_{1}}{n}
$$

by Proposition 10(a), we have the following inequality by (14)

$$
\# P>N-n+1-\varepsilon_{1}>N-n+1-\frac{N-n}{(N-n+1)(n+1)}>N-n .
$$

We have that $\# P=N-n+1$. Substituting $\# P=N-n+1$ and $d(P)=1$ in (17) we obtain that $\theta_{q}=N / n$.

Next, by Lemma 2(d), $\left(a_{\infty}\right),(11)$ and Remark 2(a) with Corollary 3(b) we have the inequality

$$
1=d(P) \geq \sum_{j \in P} \omega_{q}(j) \geq(N-n+1) \lambda_{q} \geq 1
$$

since $d(P)=1$ as is proved above, so that we have

$$
\lambda_{q}=\frac{1}{N-n+1}=\omega_{q}(j) \quad(j \in P) .
$$

As $1 \leq d\left(P_{0}\right) \leq d(P)=1$, we have $d\left(P_{0}\right)=1$. By the choice of $P_{0}$, we have the equality 


$$
\frac{1}{\# P_{0}}=\frac{d\left(P_{0}\right)}{\# P_{0}}=\lambda_{q}=\frac{1}{N-n+1},
$$

from which we have that $\# P_{0}=N-n+1$.

Corollary 4. $\lambda_{q}=\lambda_{\infty}=1 /(N-n+1)=\omega_{q}(j)\left(j \in P_{0}\right)$.

Proposition 13. (a) $P_{1}=P_{0}$ and (b) $d_{k}(q)=n$.

Proof. First we note that

$$
\theta_{q} \omega_{q}(j)=\frac{N}{n(N-n+1)}<1 \quad\left(j \in P_{0}\right)
$$

as $\theta_{q}=N / n$ (Proposition 12(c)) and $\omega_{q}(j)=1 /(N-n+1)$ for $j \in P_{0}$ (Corollary 4). Next, we prove that $P_{0} \cap P_{k}(0)=\phi$. Suppose to the contrary that $P_{0} \cap$ $P_{k}(0) \neq \phi$. As $d\left(P_{0}\right)=1$, we have $P_{0} \subset P_{k}(0)$. Then, by Propositions 9, 12(a), Corollary 4 and Lemma 2(a) we have

$$
\begin{aligned}
n-\varepsilon_{1} / \theta_{q}<d_{k}(q) & =\sum_{j \in P_{k}(0)} \omega_{q}(j)=\sum_{j \in P_{0}} \omega_{q}(j)+\sum_{j \in P_{k}(0)-P_{0}} \omega_{q}(j) \\
& \leq 1+\frac{\#\left(P_{k}(0)-P_{0}\right)}{\theta_{q}} .
\end{aligned}
$$

By Propositions 10(b), 12(a) and 12(c) the last term of this inequality is equal to

$$
1+\frac{(n-1) n}{N}
$$

so that we have the inequality

$$
\frac{(n-1)(N-n)}{n}<\varepsilon_{1}
$$

This contradicts (14). This implies that

$$
P_{0} \cap P_{k}(0)=\phi .
$$

(18) and (19) mean that $P_{0} \subset P_{1}$. By Lemma 2(b) we have the equality

$$
\begin{aligned}
q-(2 N-n+1) & =\theta_{q}\left(\sum_{j=1}^{q} \omega_{q}(j)-n-1\right) \\
& =\theta_{q}\left(\sum_{j \notin P_{k}(0) \cup P_{0}} \omega_{q}(j)+\sum_{j \in P_{k}(0) \cup P_{0}} \omega_{q}(j)-n-1\right) .
\end{aligned}
$$

Here, as $P_{k}(0) \cap P_{0}=\phi, \quad \sum_{j \in P_{k}(0)} \omega_{q}(j)=d_{k}(q) \quad$ (the definition of $d_{k}(q)$ ) and $\sum_{j \in P_{0}} \omega(j)=1$ (Proposition 12(a), Corollary 4), we have 


$$
\sum_{j \in P_{k}(0) \cup P_{0}} \omega_{q}(j)=\sum_{j \in P_{k}(0)} \omega_{q}(j)+\sum_{j \in P_{0}} \omega_{q}(j)=d_{k}(q)+1,
$$

so that we have the equality

$$
\left(\mathrm{G}_{1}\right) \quad q-(2 N-n+1)=\theta_{q} \sum_{j \notin P_{k}(0) \cup P_{0}} \omega_{q}(j)-\theta_{q}\left(n-d_{k}(q)\right) .
$$

As $P_{0} \subset P_{1}, \theta_{q} \omega_{q}(j)=1$ for $j \notin P_{k}(0) \cup P_{1}$ and $\theta_{q} \omega_{q}(j)<1$ for $j \in P_{1}$ by Lemma $2(\mathrm{a})$ and the definition of $P_{1}, \# P_{k}(0)=N$ (Proposition 10(b)), \# $P_{0}=$ $N-n+1$ (Proposition 12(a)) and $P_{k}(0) \cap P_{1}=\phi$ by the definition of $P_{1}$, we have

$$
\begin{aligned}
\left(\mathrm{G}_{2}\right) \quad \theta_{q} \sum_{j \notin P_{k}(0) \cup P_{0}} \omega_{q}(j) & =q-(2 N-n+1)-\#\left(P_{1}-P_{0}\right)+\theta_{q} \sum_{j \in P_{1}-P_{0}} \omega_{q}(j) \\
& =q-(2 N-n+1)-\sum_{j \in P_{1}-P_{0}}\left(1-\theta_{q} \omega_{q}(j)\right) .
\end{aligned}
$$

From $\left(\mathrm{G}_{1}\right)$ and $\left(\mathrm{G}_{2}\right)$ we have the equality

$$
q-(2 N-n+1)=q-(2 N-n+1)-\sum_{j \in P_{1}-P_{0}}\left(1-\theta_{q} \omega_{q}(j)\right)-\theta_{q}\left(n-d_{k}(q)\right),
$$

so that we have the equality

$$
\sum_{j \in P_{1}-P_{0}}\left(1-\theta_{q} \omega_{q}(j)\right)+\theta_{q}\left(n-d_{k}(q)\right)=0 .
$$

If either $P_{0} \varsubsetneqq P_{1}$ or $d_{k}(q)<n$ holds, we have a contradiction since $\theta_{q} \omega_{q}(j)<1$ for $j \in P_{1}$ and $d_{k}(q) \leq n$ by (15). This means that it must hold both $P_{1}=P_{0}$ and $d_{k}(q)=n$.

Proposition 14. For any $j \in P_{0}, \delta\left(\boldsymbol{a}_{j}, f\right)=1$.

Proof. Suppose to the contrary that

$$
\min _{j \in P_{0}} \delta\left(\boldsymbol{a}_{j}, f\right)=\delta<1 \text {. }
$$

Now, for any positive number $\varepsilon_{2}$ satisfying

$$
0<\varepsilon_{2}<\min \left\{\left(1-\frac{N}{n(N-n+1)}\right)(1-\delta), \frac{(N-n)\left(1-\Omega_{k}\right)}{(N-n+1)(n+1)}\right\},
$$

we choose $s \in N$ satisfying $S=\{1, \ldots, s\} \supset P_{0}, s \geq p$ and

$$
2 N-n+1-\varepsilon_{2}<\sum_{j=1}^{s} \delta\left(\boldsymbol{a}_{j}, f\right) .
$$


Note that $2 N-n+1<s<\infty$. For this $S$ we use $\theta_{s}, \omega_{s}$ and $\lambda_{s}$ instead of $\theta, \omega$ and $\lambda$ in Section 2 respectively. Then, by the choice of $s$ the following relations hold from the results obtained in this section:

(a) $\lambda_{s}=\lambda_{\infty}=1 /(N-n+1)=\omega_{s}(j)$ for $j \in P_{0}$ (Corollary 4$)$;

(b) $\theta_{s}=N / n$ (Proposition 12(c)).

By the equality (2) in the proof of Lemma 3 in [9], Lemma 3, Remark 1 and (22) we obtain

$$
\sum_{j=1}^{s}\left(1-\theta_{s} \omega_{s}(j)\right)\left(1-\delta\left(\boldsymbol{a}_{j}, f\right)\right)<\varepsilon_{2}
$$

so that for any $j \in S$

$$
\left(1-\theta_{s} \omega_{s}(j)\right)\left(1-\delta\left(\boldsymbol{a}_{j}, f\right)\right)<\varepsilon_{2} .
$$

By the definition of $\delta$, (a) and (b) given above we have the inquality

$$
\left(1-\frac{N}{n(N-n+1)}\right)(1-\delta)<\varepsilon_{2}
$$

which is a contradiction to (21). This means that $\delta=1$ and we completes the proof of this proposition.

As in Proposition 7, we have the following

Proposition 15. Any $n$ elements of $X-\left\{\boldsymbol{a}_{j} \mid j \in P_{0}\right\}$ are linearly independent.

Summarizing Propositions from 8 through 15 given above we obtain the following

THEOREM 2. Suppose that

(i) $N>n \geq 2$;

(ii) there are an infinite number of vectors $\boldsymbol{a}_{1}, \boldsymbol{a}_{2}, \ldots \in X$ satisfying $\delta\left(\boldsymbol{a}_{j}, f\right)>$ $0(j \in \boldsymbol{N})$ and

$$
\sum_{j=1}^{\infty} \delta\left(\boldsymbol{a}_{j}, f\right)=2 N-n+1 .
$$

If $\Omega_{k}<1$ for some $k(1 \leq k \leq n+1)$, then

(a) $X_{k}(0) \subset\left\{\boldsymbol{a}_{1}, \boldsymbol{a}_{2}, \ldots\right\}$ and $\# X_{k}(0)=N$;

(b) there is a subset $P$ of $N$ satisfying

$$
\# P=N-n+1, \quad d(P)=1, \quad \delta\left(\boldsymbol{a}_{j}, f\right)=1 \quad(j \in P)
$$

and

$$
X_{k}(0) \cap\left\{a_{j} \mid j \in P\right\}=\phi ;
$$

(c) any $n$ elements of $X-\left\{\boldsymbol{a}_{j} \mid j \in P\right\}$ are linearly independent. 


\section{Application}

In this section we shall apply the result obtained in Section 3 to exponential curves. For any $n+1$ distinct complex numbers $\mu_{1}, \mu_{2}, \ldots, \mu_{n+1}$ we define a holomorphic curve $f_{e}$ by

$$
f_{e}=\left[e^{\mu_{1} z}, e^{\mu_{2} z}, \ldots, e^{\mu_{n+1} z}\right] .
$$

We call it an exponential curve ([11], p. 94). It is easy to see that $f_{e}$ is transcendental and non-degenerate. We use the notations $X_{k}(0), \Omega_{k}$ etc. given in Section 1 in this section. We denote by $\boldsymbol{e}_{1}, \boldsymbol{e}_{2}, \ldots, \boldsymbol{e}_{n+1}$ the standard basis of $C^{n+1}$.

Let $D$ be the convex polygon spanned around the $n+1$ points $\mu_{1}, \mu_{2}, \ldots$, $\mu_{n+1}$ and $\ell$ the length of the polygon, where $\ell=2\left|\mu_{j}-\mu_{k}\right|$ if the polygon reduces to a segment with the endpoints $\mu_{j}$ and $\mu_{k}$.

LEMMA 8 ([11], pp. 95-98). $T\left(r, f_{e}\right)=(\ell / 2 \pi) r+O(1)$.

LEMMA 9. $\#\left\{k \mid \Omega_{k}<1 ; 1 \leq k \leq n+1\right\} \geq 2$.

Proof. (a) The case when $D$ is an $n+1$-gon.

In this case, the points $\mu_{1}, \mu_{2}, \ldots, \mu_{n+1}$ are the vertices of $D$. We number without loss of generality the vertices $\mu_{j}(j=1, \ldots, n+1)$ in asending sequence as one goes arround $D$ in the positive direction. For any $k(1 \leq k \leq n+1)$, the $n$-gon $D_{k}$ with the vertices $\mu_{1}, \ldots, \mu_{k-1}, \mu_{k+1}, \ldots, \mu_{n+1}$ is convex. Let $\ell_{k}$ be the length of the circumference of $D_{k}$. By the representation $(1)$ of $T(r, f)$ due to Cartan given in Introduction, by the definition of $t_{k}(r, f)$ and by Lemma 8 we have

$$
t_{k}\left(r, f_{e}\right)=\frac{\ell_{k}}{2 \pi} r+O(1)
$$

and so we have

$$
\Omega_{k}=\limsup _{r \rightarrow \infty} \frac{t_{k}(r, f)}{T(r, f)}=\frac{\ell_{k}}{\ell}<1
$$

since $\ell_{k}<\ell$ as is easily seen.

(b) The case when $D$ is an $m+1$-gon $(2 \leq m \leq n-1)$.

We may suppose without loss of generality that the vertices of $D$ are $\mu_{1}, \mu_{2}, \ldots, \mu_{m+1}$. The other points $\mu_{m+2}, \ldots, \mu_{n+1}$ are on the circumference of $D$ or inside $D$.

For any $k(1 \leq k \leq m+1)$, let $D_{k}$ be the convex polygon surrounding the points $\mu_{1}, \ldots, \mu_{k-1}, \mu_{k+1}, \ldots, \mu_{n+1}$ and let $\ell_{k}$ be the length of the circumference of $D_{k}$. Then as in (a), we have

$$
t_{k}\left(r, f_{e}\right)=\frac{\ell_{k}}{2 \pi} r+O(1),
$$


and so we have

$$
\Omega_{k}=\limsup _{r \rightarrow \infty} \frac{t_{k}(r, f)}{T(r, f)}=\frac{\ell_{k}}{\ell}<1
$$

since $\ell_{k}<\ell$ as is easily seen by an application of the triangle inequality.

(c) The case when $D$ reduces to a segment $L$.

We may suppose without loss of generality that

(i) $\mu_{1}$ and $\mu_{n+1}$ are the endpoints of $L$;

(ii) The points $\mu_{1}, \mu_{2}, \ldots, \mu_{n+1}$ are in ascending sequence as one goes from $\mu_{1}$ to $\mu_{n+1}$ on $L$.

Then, as in (a) we have

$$
t_{1}\left(r, f_{e}\right)=\frac{1}{\pi}\left|\mu_{2}-\mu_{n+1}\right| r+O(1), \quad t_{n+1}\left(r, f_{e}\right)=\frac{1}{\pi}\left|\mu_{1}-\mu_{n}\right| r+O(1)
$$

and

$$
T\left(r, f_{e}\right)=\frac{1}{\pi}\left|\mu_{1}-\mu_{n+1}\right| r+O(1),
$$

from which we obtain

$$
\Omega_{1}=\frac{\left|\mu_{2}-\mu_{n+1}\right|}{\left|\mu_{1}-\mu_{n+1}\right|}<1 \quad \text { and } \quad \Omega_{n+1}=\frac{\left|\mu_{1}-\mu_{n}\right|}{\left|\mu_{1}-\mu_{n+1}\right|}<1 .
$$

Lemma 10. 1) \#\{a $\left.\in X \mid \delta\left(\boldsymbol{a}, f_{e}\right)>0\right\} \leq N(n+1)$.

2) $\delta\left(\boldsymbol{a}, f_{e}\right)=1$ if and only if $\boldsymbol{a}=a \boldsymbol{e}_{k}(a \neq 0)$ for some $k(1 \leq k \leq n+1)$ and for some nonzero constant a.

Proof. 1) Let $\boldsymbol{a}=\left(a_{1}, a_{2}, \ldots, a_{n+1}\right)$ be an element of $X$ satisfying $\delta\left(\boldsymbol{a}, f_{e}\right)>$ 0 . Then, at least one of $a_{1}, a_{2}, \ldots, a_{n+1}$ is equal to zero.

In fact, suppose to the contrary that $a_{j} \neq 0(j=1, \ldots, n+1)$. Then $\boldsymbol{e}_{1}, \boldsymbol{e}_{2}, \ldots, \boldsymbol{e}_{n+1}$ and $\boldsymbol{a}$ are in general position and by Theorem $\mathrm{A}$ for $N=n$, we have

$$
\sum_{j=1}^{n+1} \delta\left(\boldsymbol{e}_{j}, f_{e}\right)+\delta\left(\boldsymbol{a}, f_{e}\right) \leq n+1,
$$

from which we have $\delta\left(\boldsymbol{a}, f_{e}\right)=0$ since $\delta\left(\boldsymbol{e}_{j}, f_{e}\right)=1(j=1, \ldots, n+1)$.

This means that

$$
\left\{\boldsymbol{a} \in X \mid \delta\left(\boldsymbol{a}, f_{e}\right)>0\right\} \subset \bigcup_{k=1}^{n+1} X_{k}(0)
$$

and as $X$ is in $N$-subgeneral position, $\# X_{k}(0) \leq N(k=1, \ldots, n+1)$. Due to these facts we reach to the fact that

$$
\#\left\{\boldsymbol{a} \in X \mid \delta\left(\boldsymbol{a}, f_{e}\right)>0\right\} \leq N(n+1) .
$$


2) If $\boldsymbol{a}=a \boldsymbol{e}_{k}(a \neq 0)$, then it is trivial that $\delta\left(\boldsymbol{a}, f_{e}\right)=1$.

Conversely, suppose that

$$
\boldsymbol{a}=a_{j_{1}} \boldsymbol{e}_{j_{1}}+\cdots+a_{j_{m}} \boldsymbol{e}_{j_{m}} \quad\left(a_{j_{1}} \neq 0, \ldots, a_{j_{m}} \neq 0 ; 2 \leq m \leq n\right) .
$$

Let

$$
g_{e}=\left[e^{\alpha_{1} z}, \ldots, e^{\alpha_{m} z}\right] \quad\left(\alpha_{p}=\mu_{j_{p}}(p=1, \ldots, m)\right) .
$$

Then, $g_{e}$ is a transcendental and non-degenerate exponential curve and by Lemma 8

$$
T\left(r, g_{e}\right)=\frac{\ell^{\prime}}{2 \pi} r+O(1),
$$

where $(0<) \ell^{\prime}(\leq \ell)$ is the length of the convex polygon spanned around the points $\alpha_{1}, \ldots, \alpha_{m}$.

As $N\left(r, \boldsymbol{a}, f_{e}\right)=N\left(r, \boldsymbol{a}, g_{e}\right)$ and

$$
\delta\left(\boldsymbol{a}, g_{e}\right)=1-\limsup _{r \rightarrow \infty} \frac{N\left(r, \boldsymbol{a}, g_{e}\right)}{T\left(r, g_{e}\right)}=0
$$

by 1) of this lemma, we have

$$
\begin{aligned}
\delta\left(\boldsymbol{a}, f_{e}\right) & =1-\limsup _{r \rightarrow \infty} \frac{N\left(r, \boldsymbol{a}, f_{e}\right)}{T\left(r, f_{e}\right)} \\
& =1-\limsup _{r \rightarrow \infty} \frac{N\left(r, \boldsymbol{a}, g_{e}\right)}{T\left(r, g_{e}\right)} \cdot \frac{T\left(r, g_{e}\right)}{T\left(r, f_{e}\right)} \\
& =1-\frac{\ell^{\prime}}{\ell}<1 .
\end{aligned}
$$

Using these lemmas we obtain the following

THEOREM 3. When $N>n \geq 2$, for any exponential curve $f_{e}$

$$
\sum_{\boldsymbol{a} \in X} \delta\left(\boldsymbol{a}, f_{e}\right)<2 N-n+1 .
$$

Proof. Suppose to the contrary that there exists an exponential curve $f_{e}$ satisfying

$$
\sum_{\boldsymbol{a} \in X} \delta\left(\boldsymbol{a}, f_{e}\right)=2 N-n+1 .
$$

Then, as the number of $\boldsymbol{a} \in X$ satisfying $\delta\left(\boldsymbol{a}, f_{e}\right)>0$ is finite by Lemma 10-1), let $\boldsymbol{a}_{1}, \ldots, \boldsymbol{a}_{q}$ be the elements of $X$ satisfying

$$
\delta\left(\boldsymbol{a}_{j}, f_{e}\right)>0 \quad(j=1, \ldots, q)
$$


and

$$
\sum_{j=1}^{q} \delta\left(\boldsymbol{a}_{j}, f_{e}\right)=2 N-n+1
$$

where $2 N-n+1 \leq q<\infty$.

(I) The case when $q=2 N-n+1$.

In this case, as $q=2 N-n+1$ and $\delta\left(\boldsymbol{a}_{j}, f_{e}\right) \leq 1$ we obtain from (23) that

$$
\delta\left(\boldsymbol{a}_{j}, f_{e}\right)=1 \quad(j=1, \ldots, 2 N-n+1) .
$$

By Lemma 10-2), for each $j=1, \ldots, 2 N-n+1$ there exists some $k(1 \leq k \leq$ $n+1)$ satisfying $\boldsymbol{a}_{j}=\alpha_{j} \boldsymbol{e}_{k}$.

Put for each $k=1, \ldots, n+1$

$$
x_{k}=\#\left\{\boldsymbol{a}_{j} \mid \boldsymbol{a}_{j}=\alpha_{j} \boldsymbol{e}_{k} ; \alpha_{j} \neq 0,1 \leq j \leq 2 N-n+1\right\} .
$$

Then, by (23) and $q=2 N-n+1$ we have

$$
\sum_{k=1}^{n+1} x_{k}=2 N-n+1 .
$$

As $\boldsymbol{a}_{1}, \boldsymbol{a}_{2}, \ldots, \boldsymbol{a}_{2 N-n+1}$ are in $N$-subgeneral position and $2 N-n+1>N+1$, it must hold that $1 \leq x_{k}$ for each $k$ and

$$
\sum_{k=1}^{n+1} x_{k}-x_{p} \leq N, \quad(p=1,2, \ldots, n+1) .
$$

Summing up $n+1$ inequalities of (25), we obtain

$$
n \sum_{k=1}^{n+1} x_{k} \leq N(n+1) \text {. }
$$

From (24) and (26) we obtain the inequality

$$
n(2 N-n+1) \leq N(n+1),
$$

from which we have the inequality

$$
(N-n)(n-1) \leq 0,
$$

which is impossible since $N>n \geq 2$.

(II) The case when $2 N-n+1<q<\infty$.

By Lemma 9 we may suppose that

$$
\Omega_{\mu}<1 \quad \text { and } \quad \Omega_{v}<1 \quad(1 \leq \mu \neq v \leq n+1) .
$$

By Theorem 1 for $k=\mu$

(a) $X_{\mu}(0) \subset\left\{\boldsymbol{a}_{1}, \ldots, \boldsymbol{a}_{q}\right\}$ and $\# X_{\mu}(0)=N$;

(b) There exists a subset $P$ of $Q=\{1,2, \ldots, q\}$ satisfying 


$$
\# P=N-n+1, \quad d(P)=1, \quad \delta\left(\boldsymbol{a}_{j}, f_{e}\right)=1 \quad(j \in P)
$$

and

$$
X_{\mu}(0) \cap\left\{\boldsymbol{a}_{j} \mid j \in P\right\}=\phi .
$$

Note that $\# P=N-n+1 \geq 2$. By Lemma 10-2) and (b) given above we obtain that

$$
\boldsymbol{a}_{j}=\beta_{j} \boldsymbol{e}_{\mu} \quad(j \in P) .
$$

This means that $\boldsymbol{a}_{j} \in X_{v}(0)(j \in P)$, and so if we choose $n$ vectors containing at least two vectors of $\left\{\boldsymbol{a}_{j} \mid j \in P\right\}$ from $X_{v}(0)$, they are linearly dependent. On the other hand, by Theorem $1(\mathrm{c})$ for $k=v$, any $n$ elements of $X_{v}(0)$ must be linearly independent. This is a contradiction.

From (I) and (II) we have that there is no exponential curve $f_{e}$ satisfying

$$
\sum_{\boldsymbol{a} \in X} \delta\left(\boldsymbol{a}, f_{e}\right)=2 N-n+1 .
$$

We complete the proof of this theorem.

Remark 3. When $n=1$, there is an example of exponential curve $f_{e}$ which satisfies (23) for any $N \geq 2$. Put $f_{e}=\left[e^{z}, e^{2 z}\right]$ and

$$
X=\left\{\boldsymbol{a}_{j}=j \boldsymbol{e}_{1}(j=1,2, \ldots, N), \boldsymbol{a}_{j}=j \boldsymbol{e}_{2}(j=N+1, N+2, \ldots, 2 N)\right\} .
$$

Then, $X$ is in $N$-subgeneral position and

$$
\sum_{j=1}^{2 N} \delta\left(\boldsymbol{a}_{j}, f_{e}\right)=2 N .
$$

Acknowledgments. The author thanks the referee for his/her valuable comments to improve the paper.

\section{REFERENCES}

[1] H. CARTAn, Sur les combinaisons linéaires de $p$ fonctions holomorphes données. Mathematica 7 (1933), 5-31.

[2] W. Chen, Defect relations for degenerate meromorphic maps. Trans. Amer. Math. Soc., 3192 (1990), 499-515.

[ 3 ] H. Fusimoto, Value distribution theory of the Gauss map of minimal surfaces in $\boldsymbol{R}^{m}$. Aspects of Math. E21, Vieweg 1993.

[ 4 ] W. K. Hayman, Meromorphic functions. Oxford at the Clarendon Press, 1964.

[5] R. Nevanlinna, Le théorème de Picard-Borel et la théorie des fonctions méromorphes. Gauthier-Villars, Paris 1929.

[6] E. I. NochKa, On the theory of meromorphic functions. Soviet Math. Dokl., 27-2 (1983), $377-381$. 
[7] N. TodA, On the fundamental inequality for non-degenerate holomorphic curves. Kodai Math. J., 20-3 (1997), 189-207.

[8] N. Toda, An improvement of the second fundamental theorem for holomorphic curves. Proceedings of the Second ISAAC Congress, edited by H. G. W. Begehr et al., Vol. 1 (2000), 501-510 (Kluwer Academic Publishers).

[9] N. TodA, On the deficiency of holomorphic curves with maximal deficiency sum. Kodai Math. J., 24-1 (2001), 134-146.

[10] N. Toda, On the deficiency of holomorphic curves with maximal deficiency sum, II. Progress in Analysis (Proceedings of the 3rd International ISAAC Congress, edited by H. G. W. Begehr et al.), Vol. 1 (2003), 287-300 (World Scientific).

[11] H. Weyl and F. J. Weyl, Meromorphic functions and analytic curves. Ann. Math. Studies 12, Princeton 1943.

Center for General Education

Aichi Institute of Technology

e-mail: toda3-302@coral.ocn.ne.jp 